CYCU-HEP-10-12

\title{
Nonet meson properties in Nambu-Jona-Lasinio model with dimensional versus cutoff regularization
}

\author{
T. Inagaki \\ Information Media Center, Hiroshima University, Higashi-Hiroshima, Hiroshima 739-8521, Japan \\ D. Kimura \\ Learning Support Center, Hiroshima Shudo University, Hiroshima, 731-3195, Japan \\ H. Kohyama \\ Institute of Physics, Academia Sinica, Taipei 115, Taiwan and \\ Physics Division, National Center for Theoretical Sciences, Hsinchu 300, Taiwan and \\ Department of Physics, Chung-Yuan Christian University, Chung-Li 32023, Taiwan \\ A. Kvinikhidze \\ A. Razmadze Mathematical Institute of Georgian Academy of Sciences, \\ M. Alexidze Str. 1, 380093 Tbilisi, Georgia
}

(Dated: March 4, 2022)

\begin{abstract}
Nambu-Jona-Lasinio (NJL) model with Kobayashi-Maskawa-'t Hooft (KMT) term is one of low energy effective theory of QCD which includes the $U_{A}(1)$ anomaly. We investigate nonet meson properties in this model with three flavors of quarks. We employ two type of regularizations the dimensional and sharp cutoff ones. The model parameters are fixed phenomenologically for each regularization. Evaluating the kaon decay constant, the $\eta$ meson mass and the topological susceptibility, we show the regularization dependence of the results and discuss the applicability of the NJL model.
\end{abstract}

PACS numbers: $11.10 \mathrm{Kk}, 12.39 .-\mathrm{x}$

\section{INTRODUCTION}

The unique QCD Lagrangian is found by imposing the local $S U_{c}(3)$ gauge symmetry, Lorentz invariance, locality and renormalizability in four space-time dimensions. It is believed to be a fundamental theory of strong interaction between quarks and gluons. One of the features of QCD dynamics is "asymptotic freedom" which legitimates perturbation theory at short distances. Unfortunately non-perturbative effects cannot be avoided in the confinement phase which takes place at low energy scale, i.e. where the QCD coupling is not small.

Nambu-Jona-Lasinio (NJL) model [1] is a well-known and often used low energy effective theory of QCD [24]. Nambu and Jona-Lasinio have introduced a fourfermion interaction to describe the attractive force between fermions. In this model, chiral symmetry is spontaneously broken by non-vanishing expectation value for a composite operator constructed by the fermion and anti-fermion fields and the fermion mass is dynamically generated. The NJL model and its generalizations are extremely useful in the study of the light meson properties at low energy.

The four-fermion interaction is a dimension six operator in four space-time dimensions therefore the model is non-renormalizable and depends on the regularization procedure. In order to regularize fermion loop integrals one usually introduces a momentum scale $\Lambda$ to cutoff integration momenta higher than $\Lambda$. Another regularization, the dimensional one, is an analytic regularization; one calculates loop integrals as analytic functions of the space-time dimensions and uses them for the value of the space-time dimensions less than four [5 8,12 ].

In the present paper we study NJL model in the dimensional regularization and compare the results with ones obtained in the sharp cutoff regularization. NJL model in both regularizations describes well the dynamical breaking of chiral symmetry and the $\pi$ and $\sigma$ meson properties in vacuum 8]. Yet at finite density (especially when the cutoff scale is close to the Fermi momentum) important contributions to the quark loop integrals are dropped in the cutoff regularization scheme [9], while dimensional regularization leads to results consistent with QCD even in the region of asymptotic freedom, therefore we find a strong regularization dependence in the extended NJL model with an attractive force in the color anti-triplet channel for a large chemical potential [12]. Such a high density state may be realized in astrophysical objects. It is expected that the model can be tested by observing the structure of dense stars.

Non-negligible regularization dependence is observed even when considering the system at $T=\mu=0$. The cutoff scale is usually taken to be lower than $\eta^{\prime}$ meson mass $\left(m_{\eta^{\prime}} \simeq 958 \mathrm{MeV}\right)$ to reproduce light meson properties [4, 13, 14]. Then the $m_{\eta^{\prime}}$ in the low energy effective theory is not well-defined quantity. $\Lambda$ is phenomenologically fixed and it is considered as a scale above which the effective model may lose its validity. Furthermore, the cutoff regularization may break some symmetry of the Lagrangian. The cutoff regularization may cause some 
unexpected effects in the system where the strange quark and $U_{A}(1)$ anomaly play an important role. Therefore we launch a plan to study the three flavor system with $U_{A}(1)$ anomaly by using the dimensional regularization.

In the present paper the extended NJL model including the Kobayashi-Maskawa-'t Hooft (KMT) term [15, 16] is regarded as a low energy effective theory of QCD with $U_{A}(1)$ anomaly. We consider three-flavor light quarks, $u, d$ and $s$, and investigate the nonet meson properties. In Sec. II. we introduce the model Lagrangian and briefly review regularization schemes. In Sec. III we calculate the meson masses, decay constants and topological susceptibility in the leading order of $1 / N_{c}$ expansion. The results depend on the regularization parameters. In Sec. IV we evaluate the kaon decay constant, the $\eta$ meson mass, $m_{\eta}$, and the topological susceptibility $\chi$ as a function of the space-time dimension $D$ associated with loop integrals. In Sec. V the dependence of physical quantities on the current up-quark mass, $m_{u}$, is studied. After phenomenologically fixing the model parameters, we discuss the two regularizations dependence of the results. This paper shows that the model Lagrangian is not satisfactory in either of regularizations. Some concluding remarks are given in Sec. VI.

\section{NJL MODEL WITH $U_{A}(1)$ ANOMALY}

The NJL model is one of the simplest models to describe the dynamical symmetry breaking. It is often used to study the light meson properties at low energy scale. Since the $U_{A}(1)$ anomaly induces the mass difference between $\eta$ and $\eta^{\prime}$ mesons, the NJL model should be extended to include the contribution from the $U_{A}(1)$ anomaly in evaluating the nonet meson system constructed out of the three flavor light quarks.

\section{A. Model set up}

Kobayashi and Maskawa have introduced an interaction written in the determinant of the composite operator constructed by quark and anti-quark fields in the nonet meson system [15]. In QCD $\theta$-term can be transformed to the determinant term by $U_{A}(1)$ transformation. Thus we can include the contribution of the $U_{A}(1)$ anomaly through the determinant term in the NJL model. In the present paper we consider the four- and six-fermion interaction invariant under $S U_{L}(3) \otimes S U_{R}(3)$ global flavor symmetry and start with the Lagrangian,

$$
\mathcal{L}_{\mathrm{NJL}}=\sum_{i, j=1}^{3} \bar{q}_{i}(i \not \partial-\hat{m})_{i j} q_{j}+\mathcal{L}_{4}+\mathcal{L}_{6},
$$

where

$$
\begin{aligned}
& \mathcal{L}_{4}=G \sum_{a=0}^{8}\left[\left(\sum_{i, j=1}^{3} \bar{q}_{i} \lambda_{a} q_{j}\right)^{2}+\left(\sum_{i, j=1}^{3} \bar{q}_{i} i \gamma_{5} \lambda_{a} q_{j}\right)^{2}\right], \\
& \mathcal{L}_{6}=-K\left[\operatorname{det} \bar{q}_{i}\left(1-\gamma_{5}\right) q_{j}+\text { h.c. }\right]
\end{aligned}
$$

the subscripts $(i, j)$ are the flavor indices, $\hat{m}$ expresses the current quark mass matrix, $\lambda_{a}$ are the Gell-Mann matrices in the flavor space, $G$ and $K$ represent the effective coupling constants for four- and six-fermion interaction, respectively. The determinant in $\mathcal{L}_{6}$ concerns the matrix elements labeled by the flavor indices, $i j$. The order of the coupling constants are supposed to be $G N_{c} \simeq O(1)$, $K N_{c}^{2} \simeq O(1)$ therefore we work in the framework of the $1 / N_{c}$ expansion. In this paper we do not care about the flavor mixing and set the mass matrix to have a diagonal form, $\hat{m}=\operatorname{diag}\left(m_{u}, m_{d}, m_{s}\right)$. The current quark mass explicitly breaks the global $S U(3)$ flavor symmetry. Below we consider the $S U(2)$ isospin symmetric case and take $m_{u}=m_{d}$ for simplicity.

The chiral condensates $\langle\bar{u} u\rangle,\langle\bar{d} d\rangle$ and $\langle\bar{s} s\rangle$ generate the constituent quark masses, $m_{u}^{*}, m_{d}^{*}$ and $m_{s}^{*}$ inside mesons. To evaluate the constituent quark mass we solve the gap equations which are derived by differentiating the thermodynamic potential with respect to $m_{u}^{*}, m_{d}^{*}$ and $m_{s}^{*}$. The gap equation is obtained in the leading order of $1 / N_{c}$ expansion [2-4],

$$
\begin{aligned}
& m_{u}^{*}=m_{d}^{*}=m_{u}+4 G\left(i \operatorname{tr}_{\mathrm{s}, \mathrm{c}} S^{u}\right)+2 K\left(i \operatorname{tr}_{\mathrm{s}, \mathrm{c}} S^{d}\right)\left(i \operatorname{tr}_{\mathrm{s}, \mathrm{c}} S^{s}\right), \\
& m_{s}^{*}=m_{s}+4 G\left(i \operatorname{tr}_{\mathrm{s}, \mathrm{c}} S^{s}\right)+2 K\left(i \operatorname{tr}_{\mathrm{s}, \mathrm{c}} S^{u}\right)\left(i \operatorname{tr}_{\mathrm{s}, \mathrm{c}} S^{d}\right),
\end{aligned}
$$

where the symbol $\operatorname{tr}_{\mathrm{s}, \mathrm{c}}$ stands for the trace in spinor and color indices. $\operatorname{tr}_{\mathrm{s}, \mathrm{c}} S^{i}$ represent the chiral condensates, $-i \operatorname{tr}_{\mathrm{s}, \mathrm{c}} S^{u} \equiv\langle\bar{u} u\rangle$ and $-i \operatorname{tr}_{\mathrm{s}, \mathrm{c}} S^{s} \equiv\langle\bar{s} s\rangle$, which are given by the trace of the quark propagator inside mesons,

$$
\begin{aligned}
-i \operatorname{tr}_{\mathrm{s}, \mathrm{c}} S^{i} & =N_{c} \operatorname{tr}_{\mathrm{s}} \int \frac{d^{D} p}{i(2 \pi)^{D}} S^{i}(p), \\
S^{i}(p) & \equiv \frac{1}{\not p-m_{i}^{*}+i \epsilon} .
\end{aligned}
$$

Here we indicate the space-time dimension for internal quark fields by $D$. Below we omit the subscripts in $\operatorname{tr}_{\mathrm{s}, \mathrm{c}}$ for notational simplicity.

\section{B. Regularization schemes}

The fermion loop integral in Eq.(6) is divergent in four space-time dimensions to obtain a finite result we have to regularize it. The four- and six-fermion interaction are written in terms of the dimension six and nine operators, respectively. Thus the operators in $\mathcal{L}_{4}$ and $\mathcal{L}_{6}$ are irrelevant in four dimensions. It means that the results 
depend on regularization procedures. Here we use two different procedures; one is the three-momentum sharp cutoff regularization and the other is the dimensional regularization.

In the three-momentum sharp cutoff method, we cut off the space momentum component integral above the scale, $\Lambda$,

$$
\int \frac{d^{D} p}{(2 \pi)^{D}} \rightarrow \int \frac{d p_{0}}{2 \pi} \int^{\Lambda} \frac{d^{3} p}{(2 \pi)^{3}}
$$

In the dimensional regularization scheme, we regularize the divergent integral with the help of analytic continuation of the integral as a function of the space-time dimension $D$ to a non-integer value less than four,

$$
\int \frac{d^{D} p}{(2 \pi)^{D}} \rightarrow \frac{2(4 \pi)^{-D / 2}}{\Gamma(D / 2)} \int_{0}^{\infty} d p p^{D-1} .
$$

This is a kind of an analytic regularization. We regard the space-time dimensions $D$ in the fermion loop integral as one of the parameters of the effective model of QCD. The dimensional regularization is applied to momentum integrals only for internal fermion lines.

The common parameters of the models considered here are the coupling constants $G$ and $K$, the current quark masses $m_{u}\left(=m_{d}\right), m_{s}$. In the cutoff scheme, the cutoff scale $\Lambda$ is one more parameter. On the other hand in the dimensional regularization, we consider the spacetime dimension $D$ as one of the model parameters. In this case we have to introduce one more parameter, the renormalization scale $M_{0}$, to obtain results with the correct mass dimension. Thus the parameters in these two regularization methods are aligned as follows,

Cutoff: $\quad G, K, m_{u}\left(=m_{d}\right), m_{s}, \Lambda$, Dimensional: $G, K, m_{u}\left(=m_{d}\right), m_{s}, D, M_{0}$.

All the parameters should be fixed phenomenologically.

\section{MESON MASS AND DECAY CONSTANT}

In this section we shall evaluate the properties of the nonet meson system. Here we calculate the meson mass, meson decay constant and topological susceptibility in the two regularizations.

\section{A. $\pi$ and $K$ masses}

First, we consider pion and kaon. The masses of these mesons are obtained by observing the pole structure in their propagators. Employing the random-phase approximation (RPA) and the $1 / N_{c}$ expansion, the meson propagators are given by $[3,4]$

$$
\Delta_{P}\left(k^{2}\right)=\frac{2 K_{\alpha}}{1-2 K_{\alpha} \Pi_{P}\left(k^{2}\right)}+\mathrm{O}\left(N_{c}^{-1}\right),
$$

where the index $\alpha$ denotes the isospin channel and $P$ stands for the meson species, $\pi$ and $K$. The flavordependent effective couplings $K_{\alpha}$ are defined by

$$
\begin{aligned}
& K_{3} \equiv G+\frac{1}{2} K i \operatorname{tr} S^{s}, \text { for } \pi^{0}, \\
& K_{6} \equiv G+\frac{1}{2} K i \operatorname{tr} S^{u}, \text { for } K^{0}, \bar{K}^{0} .
\end{aligned}
$$

In the leading order of the $1 / N_{c}$ expansion the self-energy for each meson, $P$, is given by

$$
\begin{aligned}
& \Pi_{P}\left(k^{2}\right) \delta_{\alpha \beta} \\
& =\int \frac{d^{D} p}{i(2 \pi)^{D}} \operatorname{tr}\left[\gamma_{5} T_{\alpha} S^{i}(p+k / 2) \gamma_{5} T_{\beta}^{\dagger} S^{j}(p-k / 2)\right],
\end{aligned}
$$

where the trace runs over flavor, spinor and color indices. The $S U(3)$ matrices, $T_{\alpha}$, corresponding to different channels are, $T_{3}=\lambda_{3}$ for $\pi^{0}, T_{6}=\left(\lambda_{6}+i \lambda_{7}\right) / \sqrt{2}$ for $K^{0}$ and $T_{6}^{\dagger}$ for $\bar{K}^{0}$. Thus the self-energy for $\pi^{0}, K^{0}$ and $\bar{K}^{0} \mathrm{read}$

$$
\begin{aligned}
\Pi_{\pi}\left(k^{2}=m_{\pi}^{2}\right) & =2 \Pi_{5}^{u u}\left(k^{2}=m_{\pi}^{2}\right), \\
\Pi_{K}\left(k^{2}=m_{K}^{2}\right) & =2 \Pi_{5}^{s d}\left(k^{2}=m_{K}^{2}\right),
\end{aligned}
$$

where $\Pi_{5}^{i j}\left(k^{2}\right)$ is the loop integral

$$
\begin{aligned}
\Pi_{5}^{i j}\left(k^{2}\right)= & \int \frac{d^{D} p}{i(2 \pi)^{D}} \operatorname{tr}\left[\gamma_{5} S^{i}(p+k / 2) \gamma_{5} S^{j}(p-k / 2)\right] \\
= & \frac{1}{2}\left(\frac{i \operatorname{tr} S^{i}}{m_{i}^{*}}+\frac{i \operatorname{tr} S^{j}}{m_{j}^{*}}\right) \\
& +\frac{1}{2}\left[k^{2}-\left(m_{i}^{*}-m_{j}^{*}\right)^{2}\right] I_{i j}\left(k^{2}\right)
\end{aligned}
$$

with

$$
I_{i j}\left(k^{2}\right)=\int \frac{d^{D} p}{i(2 \pi)^{D}} \frac{\operatorname{tr}_{\mathbf{s}} 1}{\left(p^{2}-m_{i}^{* 2}\right)\left[(p-k)^{2}-m_{j}^{* 2}\right]} .
$$

Here we set $\operatorname{tr}_{\mathrm{s}} 1$ to be 4 in the cutoff and $2^{D / 2}$ in the dimensional regularization schemes respectively.

Substituting the solution of the gap equations (4) and (5) in the self-energy expression for $\pi^{0}, K^{0}$ and $\bar{K}^{0}$ and evaluating the pole structure of the denominator in Eq.(91), we obtain the on-shell conditions for pion and kaon masses,

$$
\begin{aligned}
0 & =\frac{m_{u}}{m_{u}^{*}}-\left.2 K_{3} k^{2} I_{u u}\left(k^{2}\right)\right|_{k^{2}=m_{\pi}^{2}}, \\
0 & =1-\frac{m_{s}^{*}-m_{s}}{2 m_{u}^{*}}-\frac{m_{u}^{*}-m_{u}}{2 m_{s}^{*}} \\
& -2 G\left(\frac{i \operatorname{tr} S^{u}-i \operatorname{tr} S^{s}}{m_{u}^{*}}+\frac{i \operatorname{tr} S^{s}-i \operatorname{tr} S^{u}}{m_{s}^{*}}\right) \\
& -\left.2 K_{6}\left[k^{2}-\left(m_{s}^{*}-m_{u}^{*}\right)^{2}\right] I_{u s}\left(k^{2}\right)\right|_{k^{2}=m_{K}^{2}} .
\end{aligned}
$$

These equations are used to determine the values of the constituent quark masses $m_{u}^{*}$ and $m_{s}^{*}$. Because of the 
$S U(2)$ isospin symmetry the equations for the charged pion and kaon cases are the same as for the neutral ones. The QED effect is also important for the mass differences between the neutral and charged mesons. It should be noted that Eq.(18) becomes Eq.(17) in the limit $m_{s} \rightarrow$ $m_{u}$ because the $S U(3)$ flavor symmetry is restored in this limit.

\section{B. $\pi$ and $K$ decay constants}

Next, we consider the pion and kaon decay constants. The decay constant $f_{P}$ is defined by the matrix element of the axial current between meson and vacuum states,

$$
\begin{aligned}
& i k_{\mu} f_{P} \delta_{\alpha \beta} \\
& =-M_{0}^{4-D} \int \frac{d^{D} p}{(2 \pi)^{D}} \operatorname{tr}\left[\gamma_{\mu} \gamma_{5} \frac{T_{\alpha}}{2} S^{i} g_{P q q}(0) \gamma_{5} T_{\beta}^{\dagger} S^{j}\right]
\end{aligned}
$$

where we introduce a renormalization scale $M_{0}$ to define the decay constant with a correct mass dimension, $\operatorname{dim}\left(f_{P}\right)=1$ for dimensional scheme. The trace runs over flavor, spinor and color indices. The meson-to-quarkquark coupling $g_{P q q}$ is given by

$$
g_{P q q}\left(k^{2}\right)^{-2}=M_{0}^{4-D} \frac{\partial \Pi_{P}\left(k^{2}\right)}{\partial k^{2}},
$$

Inserting Eqs. (13) and (14) into Eq. (20), we obtain the pion and kaon decay constants,

$$
\begin{aligned}
f_{\pi}^{2} M_{0}^{D-4}= & m_{u}^{* 2} I_{u u}(0) \\
f_{K}^{2} M_{0}^{D-4}= & \frac{1}{J_{u s}(0)}\left[m_{u}^{*} I_{u s}(0)+\operatorname{tr}_{\mathrm{s}} 1 \cdot N_{c}\left(m_{s}^{*}-m_{u}^{*}\right)\right. \\
& \left.\times \int_{0}^{1} d x \int \frac{d^{D} p}{i(2 \pi)^{D}} \frac{x}{\left(p^{2}-L_{u s}(0)+i \epsilon\right)^{2}}\right]^{2}
\end{aligned}
$$

where $J_{u s}$ is defined by

$$
\begin{aligned}
J_{u s}\left(k^{2}\right)= & I_{i j}\left(k^{2}\right)+2 N_{c}\left(m_{s}^{*}-m_{u}^{*}\right)^{2} \\
& \times \int_{0}^{1} d x \int \frac{d^{D} p}{i(2 \pi)^{D}} \frac{x(1-x)}{\left(p^{2}-L_{u s}\left(k^{2}\right)+i \epsilon\right)^{3}} .
\end{aligned}
$$

$L_{i j}$ is defined in the appendix (see, Eq.(A3) ). As confirmed in the case with $m_{\pi}$ and $m_{K}$, Eq.(22) corresponds to Eq.(21) in the $m_{s} \rightarrow m_{u}$ limit due to the restoration of the $S U(3)$ flavor symmetry.

\section{C. $\quad \eta$ and $\eta^{\prime}$ mesons}

As is well known, the octet state, $\eta_{8}$, and the singlet state, $\eta_{0}$, are mixed in the real world. Thus the eigenstates, $\eta$ and $\eta^{\prime}$, with diagonal mass matrix are described as mixed states of $\eta_{8}$ and $\eta_{0}$. In the RPA the propagator of the $\eta-\eta^{\prime}$ system is given by [3, 4]

$$
\Delta^{+}\left(k^{2}\right)=2 K^{+}\left[1-2 K^{+} \Pi\left(k^{2}\right)\right]^{-1}
$$

where $K^{+}$and $\Pi$ are the $2 \times 2$ matrices

$$
\begin{aligned}
K^{+} & =\left(\begin{array}{ll}
K_{00} & K_{08} \\
K_{80} & K_{88}
\end{array}\right), \\
\Pi & =\left(\begin{array}{ll}
\Pi_{00} & \Pi_{08} \\
\Pi_{80} & \Pi_{88}
\end{array}\right),
\end{aligned}
$$

with

$$
\begin{aligned}
& K_{00}=G-\frac{1}{3} K\left(i \operatorname{tr} S^{s}+2 i \operatorname{tr} S^{u}\right), \\
& K_{88}=G-\frac{1}{6} K\left(i \operatorname{tr} S^{s}-4 i \operatorname{tr} S^{u}\right), \\
& K_{08}=K_{80}=-\frac{\sqrt{2}}{6} K\left(i \operatorname{tr} S^{s}-i \operatorname{tr} S^{u}\right),
\end{aligned}
$$

and

$$
\begin{aligned}
& \Pi_{00}\left(k^{2}\right)=\frac{2}{3}\left[2 \Pi_{5}^{u u}\left(k^{2}\right)+\Pi_{5}^{s s}\left(k^{2}\right)\right], \\
& \Pi_{88}\left(k^{2}\right)=\frac{2}{3}\left[\Pi_{5}^{u u}\left(k^{2}\right)+2 \Pi_{5}^{s s}\left(k^{2}\right)\right], \\
& \Pi_{08}\left(k^{2}\right)=\Pi_{80}\left(k^{2}\right)=\frac{2 \sqrt{2}}{3}\left[\Pi_{5}^{u u}\left(k^{2}\right)-\Pi_{5}^{s s}\left(k^{2}\right)\right] .
\end{aligned}
$$

To obtain the $\eta$ and $\eta^{\prime}$ meson masses we diagonalize the inverse propagator via an orthogonal transformation [4, 14. Thus the $\eta$ and $\eta^{\prime}$ meson masses are given by the solution to the following equations,

$$
\begin{gathered}
A\left(k^{2}\right)+C\left(k^{2}\right)-\sqrt{\left\{A\left(k^{2}\right)-C\left(k^{2}\right)\right\}^{2}+4\left\{B\left(k^{2}\right)\right\}^{2}}=0 \\
\text { for } k^{2}=m_{\eta}^{2}, \\
(27) \\
A\left(k^{2}\right)+C\left(k^{2}\right)+\sqrt{\left\{A\left(k^{2}\right)-C\left(k^{2}\right)\right\}^{2}+4\left\{B\left(k^{2}\right)\right\}^{2}}=0 \\
\text { for } k^{2}=m_{\eta^{\prime}}^{2},
\end{gathered}
$$

where

$$
\begin{aligned}
& A\left(k^{2}\right)=K_{88}-2 \Pi_{00}\left(k^{2}\right) \operatorname{det} K^{+}, \\
& B\left(k^{2}\right)=-K_{08}-2 \Pi_{08}\left(k^{2}\right) \operatorname{det} K^{+}, \\
& C\left(k^{2}\right)=K_{00}-2 \Pi_{88}\left(k^{2}\right) \operatorname{det} K^{+} .
\end{aligned}
$$

The mixing angle $\theta_{\eta}$ is found to be

$$
\tan \left(2 \theta_{\eta}\right)=\frac{2 B\left(k^{2}\right)}{A\left(k^{2}\right)-C\left(k^{2}\right)} .
$$

The off-diagonal matrix elements in Eq.(24) disappear and the mixing angle $\theta_{\eta}$ vanishes in the limit $m_{s} \rightarrow m_{u}$. In this limit Eq.(27) coincides with Eqs.(17) and (18) and the mass spectrum for the octet mesons degenerates. Since the $U_{A}(1)$ anomaly breaks the degeneracy between the octet and the singlet mesons, a different on-shell condition is obtained from Eq.(28). 


\section{Topological susceptibility}

To compare the QCD axial current with the NJL axial current we introduce the topological charge density $Q(x)$ [4],

$$
Q(x) \equiv \frac{g^{2}}{32 \pi^{2}} F_{\mu \nu}^{a} \tilde{F}^{a \mu \nu}=2 K \operatorname{Im}\left[\operatorname{det} \bar{q}\left(1-\gamma_{5}\right) q\right],
$$

where $g$ is the strong coupling constant of QCD and $F_{\mu \nu}^{a}$ is the field strength for gluons. The topological susceptibility $\chi$ is defined by the correlation function between the topological charge densities at different points,

$$
\chi=\int d^{4} x\langle 0|T Q(x) Q(0)| 0\rangle_{\text {connected }}
$$

It describes some global feature of QCD dynamics. In the leading order of $1 / N_{c}$ expansion it is given by [18]

$$
\begin{aligned}
\chi= & \frac{4 K^{2}}{M_{0}^{D-4}}\left(i \operatorname{tr} S^{u}\right)^{2}\left[\left(i \operatorname{tr} S^{u}\right)\left(i \operatorname{tr} S^{s}\right)\left(\frac{2 i \operatorname{tr} S^{s}}{m_{u}^{*}}+\frac{i \operatorname{tr} S^{u}}{m_{s}^{*}}\right)\right. \\
& +\left\{\frac{1}{\sqrt{6}}\left(2 i \operatorname{tr} S^{s}+i \operatorname{tr} S^{u}\right)\left(\Pi_{00}(0), \Pi_{08}(0)\right)\right. \\
& \left.+\frac{1}{\sqrt{3}}\left(i \operatorname{tr} S^{s}-i \operatorname{tr} S^{u}\right)\left(\Pi_{08}(0), \Pi_{88}(0)\right)\right\} \Delta^{+}(0) \\
& \times\left\{\frac{1}{\sqrt{6}}\left(2 i \operatorname{tr} S^{s}+i \operatorname{tr} S^{u}\right)\left(\begin{array}{c}
\Pi_{00}(0) \\
\Pi_{08}(0)
\end{array}\right)\right. \\
& \left.\left.+\frac{1}{\sqrt{3}}\left(i \operatorname{tr} S^{s}-i \operatorname{tr} S^{u}\right)\left(\begin{array}{c}
\Pi_{08}(0) \\
\Pi_{88}(0)
\end{array}\right)\right\}\right]
\end{aligned}
$$

We compare this result with the one obtained by the lattice QCD.

\section{PARAMETER SETTING IN THE DIMENSIONAL REGULARIZATION}

In this section we discuss the physical scale and the parameter setting for the model with the dimensional regularization. In the study with the help of the NJL model, parameters are usually determined by fitting the physical quantities $\left\{m_{\pi}, f_{\pi}, m_{K}, X\right\}$, where there are several choices for $X$. Here we fit the parameters by choosing $X=m_{\eta^{\prime}}$. We reproduce these four observables in the dimensional regularization scheme without fixing two of model parameters, $m_{u}$ and $D$. As for the up quark mass, $m_{u}$, we fix it by hand and test several values. The dimension, $D$, is kept to be a free parameter. Then we calculate some meson characteristics as functions of $D$. Here we evaluate $D$-dependence of some observed physical quantities: the kaon decay constant $f_{K}, \eta$ meson mass $m_{\eta}$ and topological susceptibility $\chi$.

\section{A. Parameter setting}

The model has 6 parameters $m_{u}, m_{s}, G, K, M_{0}$ and $D$, as is already mentioned in Sec. II]. Here we set $m_{u}\left(=m_{d}\right)$ at $3,4,5$ and $6 \mathrm{MeV}$, and study the region, $2<D<4$, in which an UV stable fixed point appears for $G$. Following the previous study [4], we fit the other 4 parameters $\left\{M_{0}\right.$, $\left.G, K, m_{s}\right\}$ by using the measured physical observables [17],

$$
\begin{gathered}
m_{\pi}=138 \mathrm{MeV}, m_{K}=495 \mathrm{MeV} \\
f_{\pi}=92 \mathrm{MeV}, m_{\eta^{\prime}}=958 \mathrm{MeV}
\end{gathered}
$$

These quantities are calculated by analyzing the Eqs. (17), (18), (21) and (28), respectively. We solve these equations under the constraints imposed by the gap equations for $m_{u}^{*}$ and $m_{s}^{*}$. Eliminating the term $K i \operatorname{tr} S^{s}$ from Eqs. (4) and (17), we obtain $m_{u}^{*}$ as a function of $m_{u}$ and $D$,

$$
0=\frac{m_{u}}{m_{u}^{*}}-\left.\frac{1}{2} \frac{m_{u}^{*}-m_{u}}{i \operatorname{tr} S^{u}} k^{2} I_{u u}\left(k^{2}\right)\right|_{k^{2}=m_{\pi}^{2}} .
$$

We numerically evaluate this expression to plot $m_{u}^{*}$ as a function of $D$ in Fig. 1. Inserting this $m_{u}^{*}$, function of $D$, into Eq. (21), we also describe the renormalization scale $M_{0}$ as a function of $m_{u}$ and $D$. The $D$-dependence of $M_{0}$ is shown in Fig. 2. From Figs. 11 and 2, we confirm that $m_{u}^{*}$ and $M_{0}$ in the 3 -flavor NJL model have a behavior similar to that in the 2-flavor model [8].

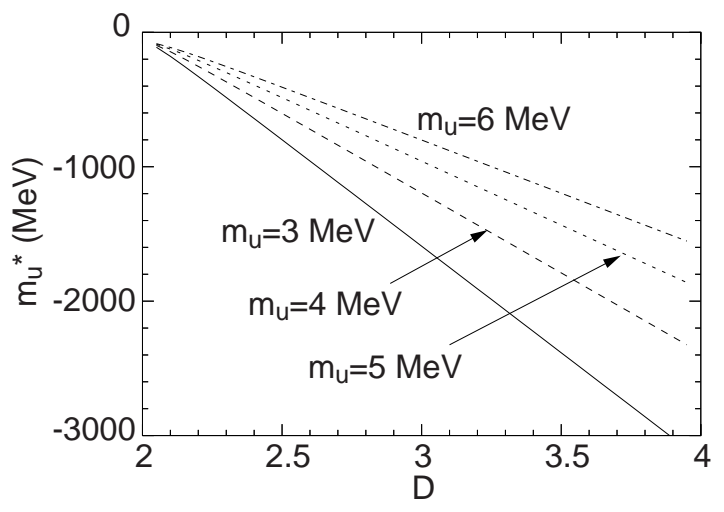

FIG. 1: The solution of the gap equation for $m_{u}^{*}$ as a function of $D$ for $m_{u}=3,4,5$ and $6 \mathrm{MeV}$.

Next we would like to fix the remaining 3 parameters $\left\{G, K, m_{s}\right\}$. From Eqs. (4), (5) and (18) the coupling constants $G$ and $K$ are obtained as functions of $m_{u}, m_{u}^{*}$ and $m_{s}^{*}$,

$$
\begin{aligned}
G\left(m_{s}^{*}\right) & =\frac{m_{u}^{*}-m_{u}}{4 i \operatorname{tr} S^{u}}-\frac{1}{2} K\left(m_{s}^{*}\right) i \operatorname{tr} S^{s}, \\
K\left(m_{s}^{*}\right) & =\frac{g\left(m_{s}^{*}\right)}{h\left(m_{s}^{*}\right)},
\end{aligned}
$$




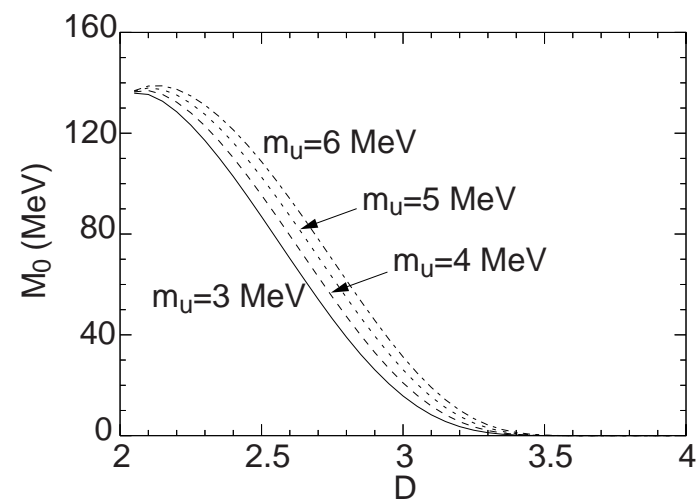

FIG. 2: The renormalization scale $M_{0}$ as a function of $D$ for $m_{u}=3,4,5$ and $6 \mathrm{MeV}$.

where $g\left(m_{s}^{*}\right)$ and $h\left(m_{s}^{*}\right)$ are given by

$$
\begin{aligned}
g\left(m_{s}^{*}\right)= & -1+\frac{m_{u}^{*}-m_{u}}{2 m_{s}^{*}} \\
& +\frac{m_{u}^{*}-m_{u}}{2 i \operatorname{tr} S^{u}}\left[\frac{i \operatorname{tr} S^{u}}{m_{u}^{*}}+\frac{i \operatorname{tr} S^{s}-i \operatorname{tr} S^{u}}{m_{s}^{*}}\right. \\
& \left.+\left\{m_{K}^{2}-\left(m_{u}^{*}-m_{s}^{*}\right)^{2}\right\} I_{u s}\left(m_{K}^{2}\right)\right], \\
h\left(m_{s}^{*}\right)= & \left(i \operatorname{tr} S^{s}-i \operatorname{tr} S^{u}\right)\left[\frac{i \operatorname{tr} S^{u}}{m_{u}^{*}}+\frac{i \operatorname{tr} S^{s}}{m_{s}^{*}}\right. \\
& \left.+\left\{m_{K}^{2}-\left(m_{u}^{*}-m_{s}^{*}\right)^{2}\right\} I_{u s}\left(m_{K}^{2}\right)\right] .
\end{aligned}
$$

Substituting Eqs. (35) and (36) into Eq. (18), we describe the current strange quark mass, $m_{s}$, as a function of $m_{u}, m_{u}^{*}$ and $m_{s}^{*}$. Then the $\eta^{\prime}$ meson mass (28) is also expressed by a function of $m_{u}, m_{u}^{*}$ and $m_{s}^{*}$. From the expression for $m_{\eta^{\prime}}$ and Eq. (34) we evaluate $m_{s}^{*}$ numerically.

The $D$-dependence of $m_{s}^{*}$ is shown in Fig. 3. We should note that qualitatively the solution for $m_{s}^{*}$ has a similar to $m_{u}^{*}$ behavior. Decreasing the dimension from four, we observe that the absolute value of $m_{s}^{*}$ goes down almost linearly. A different tendency takes place near the dimension two, a discontinuity is observed at $D \simeq 2.5$. A physical solution for $m_{s}^{*}$ does not appear, neither it is not seen for $m_{u}^{*}$ in Fig. 1.

Once $m_{s}^{*}$ is obtained, $G\left(m_{s}^{*}\right), K\left(m_{s}^{*}\right), m_{s}\left(m_{s}^{*}\right)$ are calculated by inserting the solution for $m_{s}^{*}$. The numerical results for $G, K$ and $m_{s}$ are shown in Figs. 4, 5 and 6 , respectively. In Fig. 团 we observe a similar behavior of the four-fermion coupling, $G$, to one in the 2 -flavor NJL model [8], while we do not have a consistent solution for the dimension where no solution is found for $m_{s}^{*}$. There is no analog of Fig. 5 in the 2 -flavor case. In Fig. 5 we see that $K$ gets drastically large near the dimension where a consistent solution is lost. Finally in Fig. 6 it is found that the value of the current strange quark mass $m_{s}$ is roughly constant for the dimension larger than that corresponding to the discontinuity point, but it falls down as $D$ decreases below the discontinuity point.

In Figs. 3, 4 5]and 6, we observe a discontinuity around $D \simeq 2.5$ where the behavior drastically changed. It comes from the fact that the values of the couplings $G$ and $K$ become divergent when $m_{s}^{*}$ approaches $m_{u}^{*}$. The self-energy $\Pi_{5}^{i i}\left(k^{2}=m_{\eta^{\prime}}^{2}\right)$ is also divergent at $m_{\eta^{\prime}}^{2}=$ $4 m_{i}^{* 2}$. Therefore $m_{s}^{*}$ has no physical solution around $m_{s}^{*} \simeq m_{u}^{*} \simeq m_{\eta^{\prime}} / 2$, as is numerically confirmed in Fig. 3. This is the reason why the behavior of $m_{s}^{*}$ is different from that of $m_{u}^{*}$ near the dimension two. The constituent strange quark mass, $m_{s}^{*}$, can not develop a value smaller than $m_{\eta^{\prime}} / 2$. At the discontinuity the $\eta^{\prime}$ meson propagator contains a imaginary part which corresponds to the decay width.

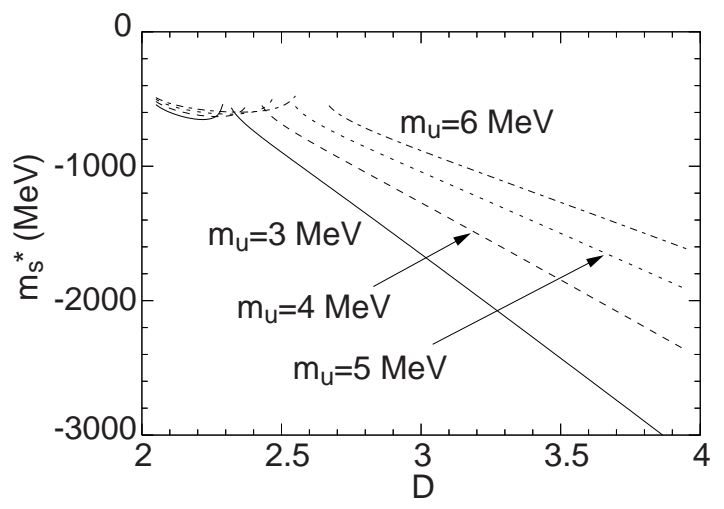

FIG. 3: The solution of the gap equation $m_{u}^{*}$ as a function of $D$ for $m_{u}=3,4,5$ and $6 \mathrm{MeV}$.

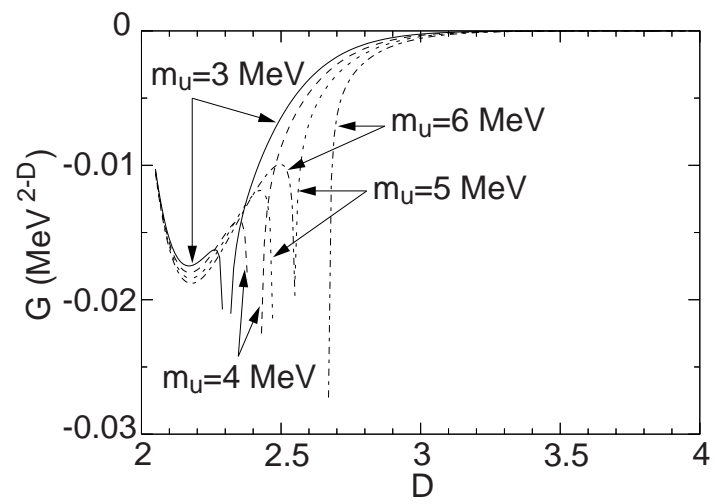

FIG. 4: The 4-fermion coupling $G$ as functions of $D$ for $m_{u}=$ $3,4,5$ and $6 \mathrm{MeV}$.

\section{B. Physical quantities}

We have fixed the model parameters in the previous subsection except for the up quark mass $m_{u}$ and the dimension, $D$. We are now ready for evaluating various meson properties as a function of $D$ for a fixed $m_{u}$. Employing the obtained parameters and the chiral condensates, $m_{u}^{*}$ and $m_{s}^{*}$, one can numerically calculate the kaon decay constant, $f_{K}$, the $\eta$ meson mass, $m_{\eta}$, and the topological susceptibility, $\chi$. The behavior of $f_{K}, m_{\eta}$ and $\chi$ are displayed in Figs. 7 8 and 9, respectively. These values 


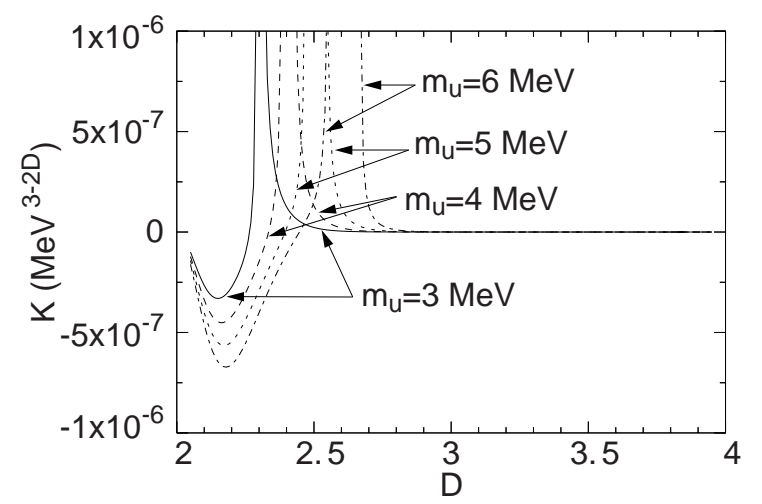

FIG. 5: The 6-fermion coupling $K$ as functions of $D$ for $m_{u}=$ $3,4,5$ and $6 \mathrm{MeV}$.

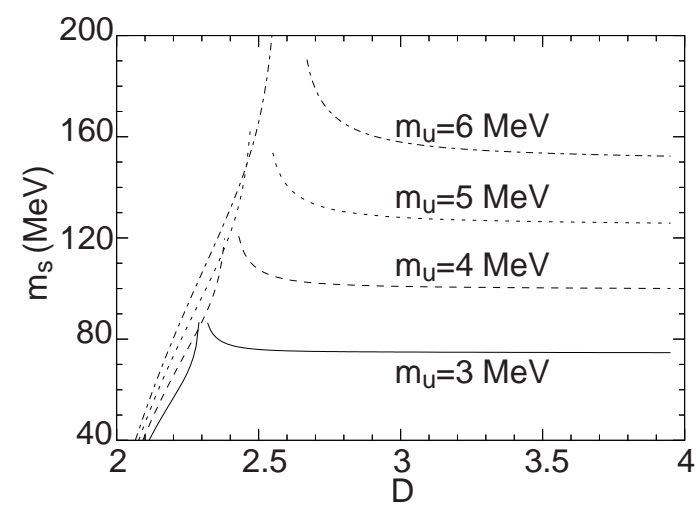

FIG. 6: The strange quark mass $m_{s}$ as a function of $D$ for $m_{u}=3,4,5$ and $6 \mathrm{MeV}$.

are roughly constant near four dimensions. They rapidly fall down as $D$ decreases near two dimensions. We again observe a discontinuity around $m_{s}^{*} \simeq m_{u}^{*} \simeq m_{\eta^{\prime}} / 2$. The topological susceptibility blows up around the discontinuity.

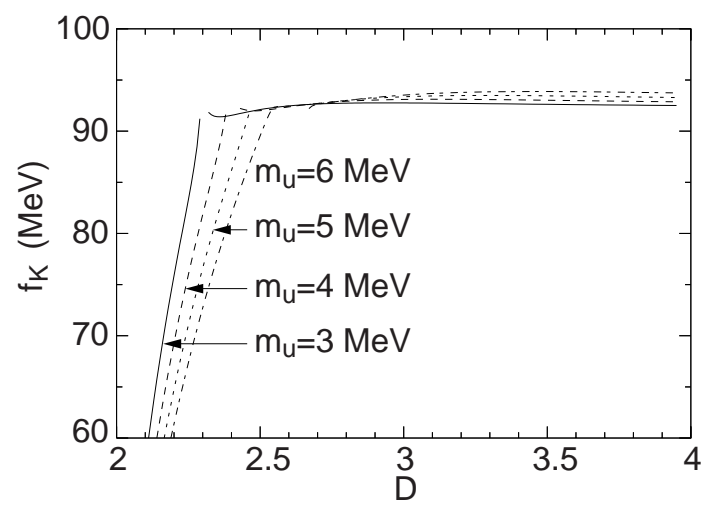

FIG. 7: The pion decay constant $f_{K}$ as a function of $D$ for $m_{u}=3,4,5$ and $6 \mathrm{MeV}$.

As is seen in Fig. 7, the decay constant $f_{K}$ is smaller than the observed one, $110 \mathrm{MeV}$, in the region, $2<D<$ 4. The model has to be improved to describe the Kaon

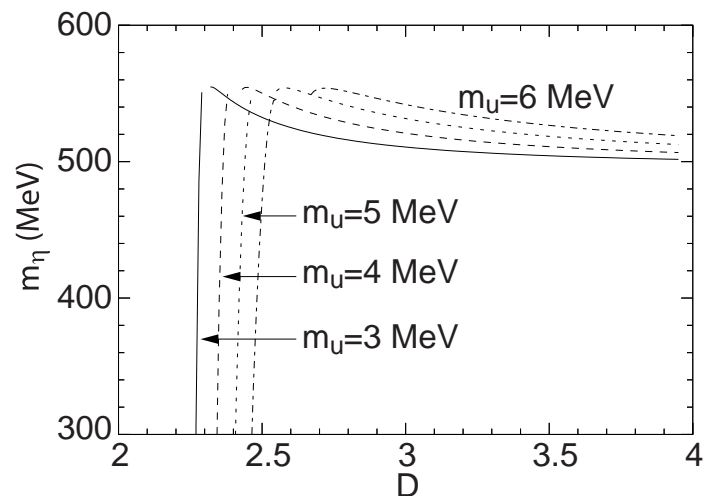

FIG. 8: The $\eta$ mass $m_{\eta}$ as a function of $D$ for $m_{u}=3,4,5$ and $6 \mathrm{MeV}$.

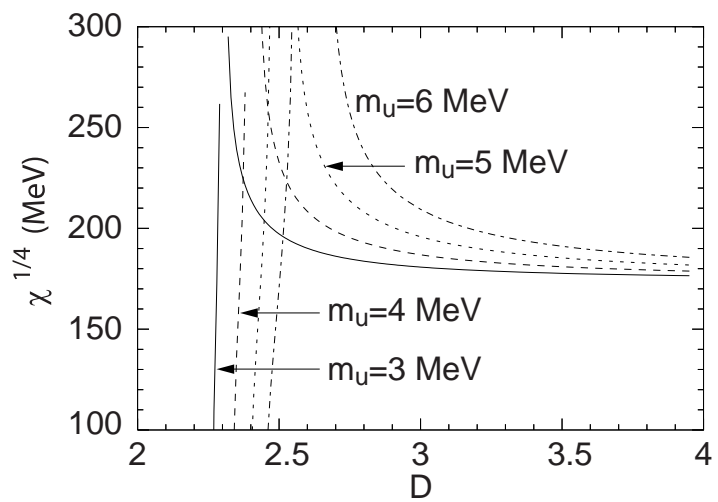

FIG. 9: The topological susceptibility $\chi$ as a function of $D$ for $m_{u}=3,4,5$ and $6 \mathrm{MeV}$.

decay. We can fit the $\eta$ meson mass to $m_{\eta}=548 \mathrm{MeV}$ by tuning the dimension of the fermion loop integrals, $D$. The topological susceptibility is calculated to be $\chi^{1 / 4}=170 \pm 7,174 \pm 7 \mathrm{MeV}$ in lattice simulations [19] and $\chi^{1 / 4}=179 \mathrm{MeV}$ in the Witten-Veneziano mass formula [20, 21]. We can also fit the topological susceptibility in the interval, $2<D<4$. Consequently the dimension for the fermion loop integrals, $D$, can be fixed to reproduce the $\eta$ meson mass or the topological susceptibility. We will discuss this matter in the next section where we need to fit an additional parameter $D$.

\section{QUARK MASS DEPENDENCE}

In the cutoff regularization all the model parameters can be fixed by fitting the physical quantities $\left\{m_{\pi}, f_{\pi}, m_{K}, m_{\eta^{\prime}}\right\}$ for a given $m_{u}$. In the dimensional regularization an an additional observable, $X^{\prime}$, needs to be fitted due to one more parameter involved in this case. Therefore, there are five physical quantities, i.e. the set of them, $\left\{m_{\pi}, f_{\pi}, m_{K}, m_{\eta^{\prime}}, X^{\prime}\right\}$, which are needed to fit all the model parameters. In this section we consider two different observables for $X^{\prime}$; one is the $\eta$ meson mass, $X^{\prime}=m_{\eta}$, another is the topological susceptibil- 
ity, $X^{\prime}=\chi$. By using the experimental/empirical values of $m_{\eta}$ and $\chi$ we can define two different parameter sets and evaluate the meson properties. Then some physical quantities are evaluated for $m_{u}=3,4,5,5.5$ and $6 \mathrm{MeV}$. We calculate the physical quantities in the both regularizations to compare them with each other for each given $m_{u}$.

\section{A. Physical quantities in the dimensional regularization}

To show the validity of the model as a low energy effective theory of QCD here we evaluate parameters of the model to reproduce the input sets $\left\{m_{\pi}, f_{\pi}, m_{K}, m_{\eta^{\prime}}, m_{\eta}\right\}$ or $\left\{m_{\pi}, f_{\pi}, m_{K}, m_{\eta^{\prime}}, \chi\right\}$ in the dimensional regularization and discuss the other (output) physical quantities. There exist two solutions to reproduce $m_{\eta}=548 \mathrm{MeV}$ for $m_{u} \lesssim 5 \mathrm{MeV}$. In Table — we show $m_{s}, f_{K}, \chi,\langle\bar{u} u\rangle_{r}$ under the condition that $m_{\eta}=548 \mathrm{MeV}$. The subscript $r$ in $\langle\bar{u} u\rangle_{r}$ stands for that the quantity is renormalized, $\langle\bar{u} u\rangle_{r}=\langle\bar{u} u\rangle M_{0}^{4-D}$. The last lines in Table [I and II show the experimental/empirical values. The light quark masses are evaluated at $1 \mathrm{GeV}$ [17]. The empirical value of $\langle\bar{u} u\rangle \simeq\langle\bar{s} s\rangle$ is evaluated by using the Gell-Mann-Oakes-Renner relation [22]. We observe that the topological susceptibility $\chi$ is always larger than the one in the lattice simulation and the Witten-Veneziano mass formula.

In the Tables II and III we fix the parameters of the model so that to reproduce $\chi^{1 / 4}=170$ or $179 \mathrm{MeV}$ and then we calculate $m_{s}, f_{K}, m_{\eta},\langle\bar{u} u\rangle_{r}$ for $m_{u}=$ $3,4,5,5.5,6 \mathrm{MeV}$. As is shown in Fig. 9] we find two solutions in both sides of the discontinuity to satisfy $\chi^{1 / 4}=179 \mathrm{MeV}$ for $m_{u} \lesssim 4 \mathrm{MeV}$. In any case, we obtain the value for the $\eta$ meson mass which is smaller than the observed one, $m_{\eta}=548 \mathrm{MeV}$.

TABLE I: Physical quantities in the dimensional regularization (in units of $\mathrm{MeV}$, except for $D$ ), $m_{\eta}$ is fixed at $548 \mathrm{MeV}$. The last line shows the experimental/empirical values.

\begin{tabular}{cccccc}
\hline \hline$m_{u}$ & $m_{s}$ & $f_{K}$ & $\chi^{1 / 4}$ & $-\langle\bar{u} u\rangle_{r}^{1 / 3}$ & $D$ \\
\hline 3.0 & 84.9 & 90.8 & 244 & 301 & 2.289 \\
3.0 & 79.0 & 91.4 & 225 & 301 & 2.372 \\
4.0 & 118 & 91.4 & 254 & 274 & 2.378 \\
4.0 & 106 & 92.1 & 225 & 273 & 2.522 \\
5.0 & 156 & 92.0 & 275 & 254 & 2.465 \\
5.0 & 134 & 92.7 & 224 & 254 & 2.687 \\
5.5 & 148 & 92.9 & 224 & 246 & 2.775 \\
6.0 & 162 & 93.2 & 224 & 239 & 2.867 \\
\hline $3.4-6.8$ & $94.5-176$ & 110 & $170-179$ & $228-287$ \\
\hline \hline
\end{tabular}

TABLE II: Physical quantities in the dimensional regularization (in units of $\mathrm{MeV}$, except for $D$ ), $\chi^{1 / 4}$ is fixed at $170 \mathrm{MeV}$. The last line shows the experimental/empirical values.

\begin{tabular}{cccccc}
\hline \hline$m_{u}$ & $m_{s}$ & $f_{K}$ & $m_{\eta}$ & $-\langle\bar{u} u\rangle_{r}^{1 / 3}$ & $D$ \\
\hline 3.0 & 77.1 & 88.4 & 481 & 302 & 2.280 \\
4.0 & 105 & 88.8 & 478 & 274 & 2.360 \\
5.0 & 134 & 89.2 & 475 & 255 & 2.434 \\
5.5 & 150 & 89.3 & 473 & 247 & 2.467 \\
6.0 & 166 & 89.5 & 471 & 240 & 2.500 \\
\hline $3.4-6.8$ & $94.5-176$ & 110 & 548 & $228-287$ & \\
\hline \hline
\end{tabular}

TABLE III: Physical quantities for the dimensional regularization (in units of $\mathrm{MeV}$, except for $D$ ), $\chi^{1 / 4}$ is fixed at $179 \mathrm{MeV}$.

\begin{tabular}{cccccc}
\hline \hline$m_{u}$ & $m_{s}$ & $f_{K}$ & $m_{\eta}$ & $-\langle\bar{u} u\rangle_{r}^{1 / 3}$ & $D$ \\
\hline 3.0 & 78.0 & 88.7 & 498 & 301 & 2.281 \\
3.0 & 74.8 & 92.7 & 507 & 300 & 3.222 \\
4.0 & 106 & 89.1 & 495 & 274 & 2.363 \\
4.0 & 100 & 92.9 & 507 & 272 & 3.895 \\
5.0 & 136 & 89.5 & 491 & 255 & 2.438 \\
5.5 & 152 & 89.7 & 489 & 247 & 2.472 \\
6.0 & 168 & 89.9 & 487 & 240 & 2.505 \\
\hline \hline
\end{tabular}

\section{B. Physical quantities in the cutoff regularization}

In this section we fix the model parameters to reproduce the measured values of Eq.(33) in the cutoff regularization for a given $m_{u}$. The quantities, $\left\{m_{s}, G, K, \Lambda\right\}$, are found following the same procedure as in the previous section. In the Table IV we show the parameters, $G, K$ and $\Lambda$ for $m_{u}=3,4,5,5.5$ and $5.87 \mathrm{MeV}$. It is observed that the cutoff scale $\Lambda$ decreases as $m_{u}$ increases. No solution is found to simultaneously satisfy Eqs. (17) and (21) for $m_{u} \gtrsim 5.87 \mathrm{MeV}$. In Table $\mathrm{V}$ the quantities, $m_{\eta}, \chi, m_{s},\langle\bar{u} u\rangle$, are shown. In the cutoff regularization the kaon decay constant, $f_{K}$, is consistent with the observed value for a smaller $m_{u}$, while the $\eta$ meson mass and the topological susceptibility are smaller than the experimental/empirical values. We obtain only smaller $\eta$ meson mass than the experimental one, similarly to the situation in the dimensional regularization with fixed topological susceptibility $\chi$.

Thus none of the regularizations can describe all of the nonet meson properties. We can fit the $\eta$ meson mass or the topological susceptibility in the dimensional regularization but we obtain only a smaller kaon decay constant in either case. In the Pauli-Villars regularization it has been found that $m_{u}=m_{d}=2.7 \mathrm{MeV}, m_{s}=92 \mathrm{MeV}$, $f_{K}=131 \mathrm{MeV}$ and $m_{\eta}=526 \mathrm{MeV}$ where the input parameters $m_{\pi}, m_{K}, f_{\pi}$ and $m_{\eta^{\prime}}$ have been used [23]. 
TABLE IV: Parameter list for cutoff scale $\Lambda$, 4-fermion coupling $G$ and 6-fermion coupling $K$.

\begin{tabular}{cccc}
\hline \hline$m_{u}(\mathrm{MeV})$ & $\Lambda(\mathrm{MeV})$ & $G \Lambda^{2}$ & $K \Lambda^{5}$ \\
\hline 3.0 & 960 & 1.55 & 8.34 \\
4.0 & 797 & 1.60 & 8.38 \\
5.0 & 682 & 1.71 & 8.77 \\
5.5 & 630 & 1.81 & 9.17 \\
5.87 & 580 & 2.09 & 10.1 \\
\hline \hline
\end{tabular}

TABLE V: Physical quantities in the cutoff regularization (in units of $\mathrm{MeV}$ ).

\begin{tabular}{cccccc}
\hline \hline$m_{u}$ & $m_{s}$ & $f_{K}$ & $m_{\eta}$ & $\chi^{1 / 4}$ & $-\langle\bar{u} u\rangle^{1 / 3}$ \\
\hline 3.0 & 89.5 & 113 & 451 & 160 & 301 \\
4.0 & 110 & 107 & 457 & 158 & 273 \\
5.0 & 128 & 101 & 473 & 160 & 253 \\
5.5 & 136 & 97.3 & 482 & 163 & 245 \\
5.87 & 139 & 93.3 & 501 & 172 & 240 \\
\hline \hline
\end{tabular}

\section{CONCLUSION}

We studied nonet meson properties in the three-flavor NJL model with the dimensional and sharp cutoff regularizations in the leading order of the $1 / N_{c}$ expansion. We employed $m_{u}, m_{\pi}, m_{K}, f_{\pi}, m_{\eta^{\prime}}$ as input parameters and fix the model parameters, $m_{s}, G, K, M_{0}$, and $m_{s}, G, K, \Lambda$ in the dimensional and cutoff regularizations, respectively. In the case of the dimensional regularization the dimension, $D$, is still a free parameter. Thus we evaluate the kaon decay constant, the $\eta$ meson mass and the topological susceptibility as a function of $D$.

The constituent up-quark mass, $m_{u}^{*}$, and the renormalization scale $M_{0}$ behave in a similar to the two-flavor case way. No consistent solution for $m_{s}^{*}$ is found and the effective coupling, $G$ and $K$, are divergent around $m_{s}^{*} \simeq m_{u}^{*} \simeq m_{\eta^{\prime}} / 2$. Below this region $\eta^{\prime}$ meson propagator develops an imaginary part. The kaon decay constant, $f_{K}$, is smaller than the observed value, $110 \mathrm{MeV}$, for $2<D<4$.

We found that $m_{s}, f_{K}, m_{\eta}, \chi$ are almost constant near four dimensions. Fitting the parameters at lower dimension and then taking the four dimensional limit, obtained values for $m_{s}, f_{K}, m_{\eta}, \chi$ are not divergent. The results do not depend on the regularization parameter. It bring us an interesting idea of making a regularization independent prediction in the NJL model.

We have evaluated the physical quantities, $m_{s}, f_{K}, \chi$, $m_{\eta}$ and $\langle\bar{u} u\rangle$, in both the dimensional and the cutoff regularizations for a fixed $m_{u}$. In the dimensional regularization the topological susceptibility, $\chi$, develops a larger value than the one obtained in the lattice simulation and Witten-Veneziano mass formula for the fixed $m_{\eta}$. On the other hand, the $\eta$ meson acquires a smaller mass than the observed one for the fixed $\chi$.

In the cutoff regularization the cutoff scale significantly depends on $m_{u}$; the cutoff $\Lambda$ increases with decreasing $m_{u}$. It is interesting to note that the coupling constants become smaller when one takes the larger cutoff, which is consistent with the renormalization group argument where the coupling strength becomes smaller with increasing the energy-scale. This cutoff effect is also confirmed numerically in the NJL model through changing the cutoff in the temporal direction [24].

In the Tables. III III and $\mathrm{V}$ some difficulty is seen in tuning $f_{K}, m_{\eta}$ and $\chi$ simultaneously. Thus, this paper shows that the model based on the Lagrangian (1) is not satisfactory in either of regularizations. It teaches us that new terms should be added to the Lagrangian to improve the model at least in one of the regularizations. Especially, we are interested in including vector type and multi-fermion interactions and hope to report on the problem in future.

\section{Acknowledgments}

The authors would like to thank Y. Hoshino and Y. Kitadono for fruitful discussions. HK is supported by the grant NSC-99-2811-M-033-017 from National Science Council (NSC) of Taiwan. Discussions during 2009 International "Workshop on Strong Coupling Gauge Theories in LHC Era" (SCGT 09) and the YIPQS international workshop on "New Frontiers in QCD 2010", were useful to complete this work.

\section{Appendix A: Integrals}

In this section, we demonstrate several integrals both in the cutoff and dimensional regularization schemes which are required in the section $\amalg$ and $\amalg$.

\section{The cutoff regularization}

The chiral condensates $i \operatorname{tr} S^{i}$ in Eq.(6) are important quantities because they concern gap equations. They take the following form after the integration is performed,

$$
\begin{aligned}
i \operatorname{tr}_{\mathrm{s}, \mathrm{c}} S^{i}= & \frac{N_{c}}{2 \pi^{2}} m_{i}^{*}\left\{\Lambda \sqrt{\Lambda^{2}+m_{i}^{* 2}}\right. \\
& \left.-m_{i}^{* 2} \ln \frac{\Lambda+\sqrt{\Lambda^{2}+m_{i}^{* 2}}}{\sqrt{m_{i}^{* 2}}}\right\} .
\end{aligned}
$$

The next integral to carry out is $I_{i j}\left(k^{2}\right)$ in Eq. (16) which determines self-energies of mesons, $\Pi_{P}$, Eq.(12). It takes 
the form

$$
\begin{aligned}
I_{i j}= & \frac{N_{c}}{8 \pi^{2}} \int_{0}^{1} d x\left\{-\frac{\Lambda}{\sqrt{\Lambda^{2}+L_{i j}\left(k^{2}\right)}}\right. \\
& \left.+\ln \frac{\Lambda+\sqrt{\Lambda^{2}+L_{i j}\left(k^{2}\right)}}{\left|\sqrt{L_{i j}\left(k^{2}\right)}\right|}\right\},
\end{aligned}
$$

where

$$
L_{i j}\left(k^{2}\right)=m_{i}^{* 2}-\left(m_{i}^{* 2}-m_{j}^{* 2}\right) x-k^{2} x(1-x) .
$$

Finally, Eqs. (22) and (23) involved in the derivation of the kaon decay constant are calculated as

$$
\begin{aligned}
f_{K}^{2}= & \frac{4}{J_{u s}(0)}\left[m_{u}^{*} I_{u s}(0)-\frac{N_{c}}{8 \pi^{2}}\left(m_{s}^{*}-m_{u}^{*}\right)\right. \\
& \times \int_{0}^{1} d x x\left\{\frac{\Lambda}{\sqrt{\Lambda^{2}+L_{u s}(0)}}\right. \\
& \left.\left.-\ln \frac{\Lambda+\sqrt{\Lambda^{2}+L_{u s}(0)}}{\sqrt{L_{u s}(0)}}\right\}\right]^{2}, \\
J_{u s}\left(k^{2}\right)= & I_{u s}\left(k^{2}\right)-\frac{N_{c}}{16 \pi^{2}} \Lambda^{3}\left(m_{s}^{*}-m_{u}^{*}\right)^{2} \\
& \times \int_{0}^{1} d x \frac{x(1-x)}{L_{u s}\left(k^{2}\right)\left[\Lambda^{2}+L_{u s}\left(k^{2}\right)\right]^{3 / 2}} .
\end{aligned}
$$

\section{The dimensional regularization}

The corresponding integrals in the dimensional regularization are performed as

$$
\begin{aligned}
i \operatorname{tr}_{\mathrm{s}, \mathrm{c}} S^{i}= & \frac{N_{c}}{(2 \pi)^{D / 2}} \Gamma\left(1-\frac{D}{2}\right) m_{i}^{*}\left(m_{i}^{* 2}\right)^{D / 2-1}, \\
f_{K}^{2}= & \frac{2^{D / 2}}{M_{0}^{D-4} J_{u s}(0)}\left[m_{u}^{*} I_{u s}(0)+\frac{N_{c}}{(4 \pi)^{D / 2}} \Gamma\left(2-\frac{D}{2}\right)\right. \\
& \left.\times\left(m_{s}^{*}-m_{u}^{*}\right) \int_{0}^{1} d x x L_{u s}(0)^{D / 2-2}\right]^{2}, \\
J_{u s}= & I_{u s}\left(k^{2}\right)-\frac{N_{c}}{(4 \pi)^{D / 2}} \Gamma\left(3-\frac{D}{2}\right)\left(m_{s}^{*}-m_{u}^{*}\right)^{2} \\
& \times \int_{0}^{1} d x x(1-x) L_{u s}\left(k^{2}\right)^{D / 2-3} .
\end{aligned}
$$

Regarding $I_{i j}$, we have different expressions depending on the value of $k^{2}$;

$$
I_{i j}=\frac{N_{c}}{(4 \pi)^{D / 2}} \Gamma\left(2-\frac{D}{2}\right) \int_{0}^{1} d x L_{i j}^{D / 2-2}\left(k^{2}\right),
$$

$$
\begin{aligned}
& I_{i j}\left(k^{2}\right)=\frac{N_{c}}{(4 \pi)^{D / 2}} \Gamma\left(2-\frac{D}{2}\right)\left[\frac{2}{D-2} \nu_{i j}^{D / 2-2}\right. \\
& \times\left\{a_{-}^{D / 2-1} F\left(2-\frac{D}{2}, \frac{D}{2}-1, \frac{D}{2} ;-\frac{a_{-}}{a_{+}-a_{-}}\right)\right. \\
& \left.+\left(1-a_{+}\right)^{D / 2-1} F\left(2-\frac{D}{2}, \frac{D}{2}-1, \frac{D}{2} ;-\frac{1-a_{+}}{a_{+}-a_{-}}\right)\right\} \\
& \left.+e^{i \pi(2-D / 2)}\left(k^{2}\right)^{1-D / 2} \nu_{i j}^{D-3} B\left(\frac{D}{2}-1, \frac{D}{2}-1\right)\right]
\end{aligned}
$$

for $0<k^{2}<\left(m_{i}^{*}-m_{j}^{*}\right)^{2},\left(m_{i}^{*}+m_{j}^{*}\right)^{2}<k^{2}$, respectively. $F$ denotes the hypergeometric function. In Eq. (A10) we introduce the quantities $a_{ \pm}$and $\nu_{i j}$ which are defined by

$$
\begin{aligned}
& a_{ \pm}=\frac{k^{2}+\left(m_{i}^{* 2}-m_{j}^{* 2}\right) \pm \nu_{i j}}{2 k^{2}} \\
& \nu_{i j}=\sqrt{k^{4}-2 k^{2}\left(m_{i}^{* 2}+m_{j}^{* 2}\right)+\left(m_{i}^{* 2}-m_{j}^{* 2}\right)^{2}} .
\end{aligned}
$$

(1992).

[1] Y. Nambu and G. Jona-Lasinio, Phys. Rev. 122, 345 (1961); ibid. 124, 246 (1961).

[2] U. Vogl and W. Weise, Prog. Part. Nucl. Phys. 27, 195 (1991)

[3] S.P. Klevansky, Rev. Mod. Phys. 64, 649 (1992).

[4] T. Hatsuda and T. Kunihiro, Phys. Rept. 247, 221 (1994).

[5] S. Krewald and K. Nakayama, Ann. Phys. 216, 201

[6] T. Inagaki, T. Kouno and T. Muta, Int. J. Mod. Phys. A 10, 2241 (1995).

[7] R.G. Jafarov, and V.E. Rochev Russ. Phys. J. 49, 712 (2006).

[8] T. Inagaki and D. Kimura and A. Kvinikhidze, Phys. Rev. D 77, 116004 (2008).

[9] This might be the case only in the sharp cutoff regulariza- 
tion. Some form of the smooth cut-off (or some non-local extension of the NJL model, like, for example, [10, 11]) may lead to results close to ones obtained in the dimensional regularization.

[10] R.S. Plant and M.C. Birse, Nucl. Phys. A 628, 607 (1998); ibid. A 703, 717 (2002).

[11] T. Hell, S. Roessner, M. Cristoforetti and W. Weise, Phys. Rev. D 79, 014022 (2009); ibid. D 81, 074034 (2010).

[12] T. Fujihara, D. Kimura, T. Inagaki and A. Kvinikhidze Phys. Rev. D 79, 096008 (2009).

[13] M. Lutz, S. Klimt and W. Weise, Nucl. Phys. A 542, 521 (1992).

[14] P. Rehberg, S.P. Klevansky and J. Hufner, Phys. Rev. C 53, 410 (1996).

[15] M. Kobayashi and T. Maskawa, Prog. Theor. Phys. 44, 1422 (1970); M. Kobayashi, H. Kondo and T. Maskawa, Prog. Theor. Phys. 45, 1955 (1971).

[16] G. 't Hooft, Phys. Rev. D 14, 3432 (1976); Erratum-ibid.
D 18, 2199 (1978); Phys. Rept. 142, 357 (1986).

[17] C. Amsler et al. (Particle Data Group), Physics Letters B 667, 1 (2008) and 2009 partial update for the 2010 edition.

[18] K. Fukushima, K. Ohnishi, K. Ohta, Phys. Rev. C 63, 045203 (2001).

[19] B. Alles, M. D'Elia and A. Di Giacomo, Nucl. Phys. B 494, 281 (1997); Erratum-ibid. B 679, 397 (2004).

[20] E. Witten, Nucl. Phys. B 156, 269 (1979).

[21] G. Veneziano, Nucl. Phys. B 159, 213 (1979).

[22] M. Gell-Mann, R.J. Oakes and B. Renner, Phys. Rev. 175, 2195 (1968).

[23] A.A. Osipov, A.H. Blin and B. Hiller, arXiv:hep-ph/0410148 A.A. Osipov, H. Hansen and B. Hiller, Nucl. Phys. A 745, 81 (2004).

[24] J.W. Chen, K. Fukushima, H. Kohyama, K. Ohnishi and U. Raha, Phys. Rev. D 81, 071501 (2010). 